\title{
ADUBAÇÃO FOSFATADA EM SISTEMA DE FORRAGEM SOLTEIRA E CONSORCIADA COM LEGUMINOSAS EM RAZÃO DO PISOTEIO ANIMAL
}

Caroline Honorato Rocha', Amarildo Francisquini Junior', Carlos Sérgio Tiritan'1, Douglas Celestino Junior', Tiago Aranda Catuchi', Juliano Carlos Calonego ${ }^{2}$

'Universidade do Oeste Paulista - UNOESTE, Presidente Prudente, SP. ${ }^{2}$ Universidade Estadual Paulista - UNESP, Botucatu, SP. E- mail: honorato-carol@hotmail.com

\section{RESUMO}

O objetivo deste trabalho foi avaliar o efeito da resistência à penetração do solo antes e após a saída dos animais. O experimento foi conduzido na Fazenda experimental da Universidade do Oeste Paulista - UNOESTE. Para realizar a avaliação de compactação do solo, foi utilizado o penetrômetro de impacto, sendo feito antes e após a entrada dos animais em razão de adubação fosfatada e sistema de forragens. A coleta de dados foi feita em um ponto a cada subparcela. Foi avaliado também a quantidade de matéria seca de raiz, através da coleta com trado tipo caneca, a amostra foi lava, passada em uma peneira de malha $1 \mathrm{~mm}$ e levada par a estufa $65^{\circ} \mathrm{C}$. A presença de animais na área não favoreceu a compactação do solo.

Palavras - chave: Forrageiras. Solo. Sistemas. Fontes.

\section{COMPOSITION OF THE SOIL IN A FORAGE SYSTEM FOR SOLEIRA AND CONSORCED WITH LEGUMES IN ANIMAL RATING}

\begin{abstract}
The objective of this work was to evaluate the effect of resistance to soil penetration before and after the animals exit. The experiment was conducted at the Experimental Farm of Universidade do Oeste Paulista - UNOESTE. In order to carry out the soil compaction evaluation, the impact penetrometer was used, before and after the entrance of the animals due to phosphate fertilization and fodder system. Data were collected at one point at each subplot. It was also evaluated the amount of dry matter of root, through the collection with mug type, the sample was washed, passed in a sieve of mesh $1 \mathrm{~mm}$ and taken to the oven $65^{\circ} \mathrm{C}$. The presence of animals in the area did not favor soil compaction.
\end{abstract}

Keywords: Forages. Ground. Systems. Sources

\section{INTRODUÇÃO}

Embora existam propriedades com tecnologia e manejo adequado das pastagens para elevar os indicie de produtividades de carne por unidade de área, mais de $60 \%$ encontra-se em processo de degradação e/ou degradadas. Essa degradação ocorre devido o manejo incorreto, não respeitando as exigências edafoclimáticas da cultivar, sem atender principalmente a altura de pastejo, carga animal e período de descanso, favorecendo assim a compactação do solo (CECATO et al., 2014).

A possibilidade de compactação do solo causada pelo pisoteio animal vem preocupando cada vez mais os produtores. Esta compactação influencia no crescimento vegetativo das plantas que prejudicando assim seu desenvolvimento, isso faz com que aumente a susceptibilidade do solo favorecendo a erosão, má infiltração da água e o crescimento radicular. O superpastejo é um 
dos fatores que provocam a compactação através perda de cobertura do solo, causada por altas taxas de lotação e pisoteio excessivo dos animais (MARCHÃO et al., 2007).

O penetrômetro de impacto é um aparelho de baixo custo, que não requer calibragem frequente e os resultados não dependem do operador, ele caracteriza a compactação causada pelo uso e manejo do solo (TORMENA; ROLOFF, 1996). Segundo Herrick e Jones (2002) o penetrômetro de impacto é recomendado para todas as aplicações, ele registra a penetração por unidade de profundidade, com isso o penetrômetro de impacto e estático não pode ser comparado, pelas diferenças na forma do registro.

A utilização de alternativas para minimizar a compactação do solo causada pelo manejo inadequado da área é o consórcio entre gramíneas e leguminosas que apresentem palatabilidade animal e raízes rigorosas (CARVALHO e PIRES, 2008). Uma das leguminosas forrageiras que vem sendo utilizada na consorciação é o feijão guandu, além de conter um ótimo teor de proteína, possui um sistema radicular profundo capaz de descompactar o solo e realizar ciclagem de nutrientes (AZEVEDO et al., 2007).

Portanto esse presente trabalho tem como objetivo avaliar o efeito da resistência a penetração do solo antes e após a saída dos animais em razão de adubação fosfatada e sistema de forragens.

\section{METODOLOGIA}

O experimento foi conduzido na Fazenda Experimental da Universidade do Oeste Paulista UNOESTE, em Presidente Bernardes - SP. O solo desta área é caracterizado como argissolo vermelho distroférrico, isto é, apresenta maior acúmulo de argila em profundidade (horizonte b) em relação á camada superficial do solo (EMBRAPA, 2006). O clima da região, segundo a classificação de Koppen é do tipo Cwa, onde as temperaturas médias são de 25ㄷ e os índices pluviométricos variam em determinadas épocas do ano, sendo o verão mais chuvoso e o inverno mais seco.

O delineamento experimental empregado foi em blocos casualizados em esquema fatorial com diferentes tipos de manejo de pastagens e distintas fontes de fósforo $(3 \times 3)$ em parcelas subdivididas, com quatro repetições.

As parcelas foram constituídas por três sistemas de manejo isto é, um sistema de plantio solteiro e dois sistemas de pastagens consorciadas com leguminosas:

- Sistema solteiro 01 = Panicum maximum cv. mombaça com ausência de adubação nitrogenada de cobertura;

- Consórcio 01 = Panicum maximum cv. mombaça consorciada com Java (Macrotyloma axilare $\mathrm{cv}$.$) ;$

- Consórcio 02 = Panicum maximum cv. mombaça consorciada com feijão- guandu anão (Cajanus cajan).

As subparcelas foram compostas pelas seguintes fontes de adubação fosfatadas:

- Controle: Ausência de adubação fosfatada (Sem P);

- Fonte 01: Super fosfato triplo - SFT (solúvel);

- Fonte 02: Fosfato natural bayóvar (reativo);

Cada parcela experimental se constituiu por uma área de $3.750 \mathrm{~m}^{2}$, já as subparcelas foram dispostas em áreas individuais de $937 \mathrm{~m}^{2}$.

Em Outubro de 2014 foi realizada a caracterização química do solo (Tabela 1), para tanto foram coletados amostras de solo nas camadas de 0-20 e 20-40 cm de profundidade. As amostras foram secas ao ar e analisadas quanto ás seguintes características: $\mathrm{pH}\left(\mathrm{CaCl}_{2}\right)$, matéria orgânica, base trocáveis, alumínio, hidrogênio extraíveis, fósforo disponível, foram calculadas a capacidade de troca de cátion (CTC) e a saturação por base (V\%), conforme metodologia proposta por Raij et al. (2001). 
A correção da acidez do solo e adubação fosfatada foi recomendada de acordo com a necessidade da área experimental, sendo aplicadas $2 \mathrm{t} \mathrm{ha}^{-1}$ de calcário dolomítico para elevar a saturação de base do solo para $70 \%$, e $1 \mathrm{t} \mathrm{ha}^{-1}$ de gesso agrícola para o fornecimento de enxofre. A calagem e gessagem foram feitas em dezembro de 2014, incorporadas com grade pesada na profundidade de $40 \mathrm{~cm}$.

A aplicação de fósforo na área foi a lanço com a distribuição do fertilizante no modelo Vincon regulada para a dose de $100 \mathrm{~kg} \mathrm{ha}^{-1} \mathrm{P}_{2} \mathrm{O}_{5}$, e incorporada com grade niveladora após a distribuição dos fertilizantes, sendo três combinações de fósforo como: ausência de fósforo, fósforo solúvel (SPT 48\% $\mathrm{P}_{2} \mathrm{O}_{5}$ ) e fósforo natural reativo (bayóvar $29 \% \mathrm{P}_{2} \mathrm{O}_{5}$ ), aplicado 30 dias antes da semeadura.

Tabela 1. Características químicas do solo nas profundidades $0-0,20$ e $0,20-0,40 \mathrm{~m}$, determinadas em dezembro de 2014.

\begin{tabular}{|c|c|c|c|c|c|c|c|c|c|}
\hline Profundidade & $\mathrm{pH}\left(\mathrm{CaCl}_{2}\right)$ & M.O. & $P_{\text {resina }}$ & $\mathrm{H}+\mathrm{Al}$ & K & $\mathrm{Ca}$ & $\mathrm{Mg}$ & СTC & v \\
\hline$(m)$ & & $\left(\mathrm{g} \mathrm{dm}^{-3}\right)$ & $\left(\mathrm{mg} \mathrm{dm}^{-3}\right)$ & & & $\mathrm{ol}_{\mathrm{c}} \mathrm{dm}^{3}$ ) & & & $\%$ \\
\hline $0-0,20$ & 5,2 & 18,6 & 3,1 & 21,8 & 0,8 & 10,0 & 7,9 & 40,5 & 46,3 \\
\hline $0,20-0,40$ & 5,1 & 15,2 & 2,8 & 21,8 & 0,7 & 10,1 & 6,7 & 39,3 & 44,6 \\
\hline
\end{tabular}

A semeadura das culturas foi realizada no dia 14 de março de 2015, o plantio do Panicum maximum cv. Mombaça foi feito com distribuidora de sementes modelo Show (marca Matsuda) regulada para $6 \mathrm{~kg} \mathrm{ha}^{-1}$ de sementes com VC $80 \%$. As leguminosas (feijão guandú e java) foram semeadas com a semeadora Case $\mathrm{IH}$, no espaçamento entre linhas de 0,90 $\mathrm{m}$, regulada para distribuir 15 e 3 ha $^{-1}$ de feijão guandú e java, respectivamente.

A medição de compactação foi realizada com auxílio de um penetrômetro de impacto modelo Stolf (STOLF et al., 1983; STOLF, 1991). As medidas foram realizadas antes da entrada dos animais e após a saída, durante todo o período experimental. Foi mensurado um ponto em cada subparcela, e nos sistemas de forragens consorciadas o ponto de coleta será $10 \mathrm{~cm}$ da linha da leguminosa.

A coleta foi realizada com trado tipo caneca, contendo volume conhecido, sendo que o solo com as raízes foi lavado com água corrente e passado em peneira com malha de $1 \mathrm{~mm}$ para separação das raízes, que foram secas em estufa a $65^{\circ} \mathrm{C}$ e pesadas.

Os dados foram submetidos á Análise de Variância e os valores médios comparados através do teste Tukey $(p<0,05)$, utilizando-se o programa estatístico SISVAR (FERREIRA, 2000).

\section{RESULTADOS}

Na tabela 2 os sistemas de forragens (SF) nas camadas mais superficiais até $35 \mathrm{~cm}$ não houve diferença significativa. Porém nas profundidades de $35 \mathrm{~cm}$ até $50 \mathrm{~cm}$ houve diferença no consórcio de Mombaça + java, pode ser explicado pelo sincronismo das raízes da gramínea com a leguminosa. $E$ para fontes de $P$ não apresentou diferença significativa. Houve interação entre SF e fontes de $P$ nas camadas de $15 \mathrm{~cm}$ e de $25 \mathrm{~cm}$ a $50 \mathrm{~cm}$. 
Tabela 2. Analise de compactação do solo nos sistemas de forragem (SF) e fontes de P. Presidente Bernardes-SP, antes da entrada dos animais.

\begin{tabular}{|c|c|c|c|c|c|c|c|c|c|c|c|}
\hline \multirow[t]{2}{*}{$\mathrm{SF}^{(1)}$} & 2,5 & 7,5 & 12,5 & 17,5 & 22,5 & 27,5 & 32,5 & 37,5 & 42,5 & 47,5 & 52,5 \\
\hline & \multicolumn{11}{|c|}{ Profundidade $(\mathrm{cm})$} \\
\hline$M-N$ & $2,0 a$ & $2,3 a$ & $3,4 a$ & $4,1 a$ & $4,4 a$ & $4,4 a$ & $5,1 b$ & $4,9 a$ & $4,2 b$ & $3,9 a$ & $3,7 b$ \\
\hline$M+J$ & $1,9 a$ & $2,2 a$ & $2,6 a$ & $2,9 a$ & $2,9 a$ & $2,8 a$ & $2,9 a$ & $2,8 b$ & $2,7 a$ & $2,7 a$ & $2,5 a$ \\
\hline$M+G$ & $2,3 a$ & $2,9 a$ & $4,0 a$ & $4,3 a$ & $4,5 a$ & $4,3 a$ & $4,3 a b$ & $4,2 a b$ & $3,9 a b$ & $3,6 a$ & $3,5 a b$ \\
\hline \multicolumn{12}{|l|}{ Fontes de P } \\
\hline$-P$ & $2,1 a$ & $2,6 a$ & $3,5 a$ & $3,8 a$ & $3,9 a$ & $4,3 a$ & $4,6 a$ & $4,5 b$ & $3,9 a$ & $3,8 a$ & $3,4 a$ \\
\hline Bayóvar & $2,0 a$ & $2,3 a$ & $3,3 a$ & $4,0 a$ & $4,3 a$ & $3,8 a$ & $3,6 a$ & $3,4 a$ & $3,4 a$ & $3,0 a$ & $3,0 a$ \\
\hline SFT & $2,0 a$ & $2,5 a$ & $3,3 a$ & $3,5 a$ & $3,6 a$ & $3,6 a$ & $4,0 a$ & $3,9 a b$ & $3,6 a$ & $3,4 a$ & $3,3 a$ \\
\hline$S F \times P$ & ns & ns & ns & $*$ & ns & ns & ns & $* *$ & $*$ & $*$ & $*$ \\
\hline$C V_{\text {parcela }}$ & 35,4 & 46,4 & 56,4 & 53,0 & 39,9 & 39,6 & 32,2 & 29,8 & 32,3 & 28,8 & 23,1 \\
\hline $\mathrm{CV}_{\text {subparcela }}$ & 26,7 & 26,4 & 49,2 & 37,7 & 47,5 & 30,7 & 34,2 & 22,9 & 22,0 & 22,7 & 25,3 \\
\hline
\end{tabular}

Médias seguidas de letras distintas na coluna, dentro do fator SF e fontes de $\mathrm{P}$, diferem entre si pelo teste Tukey a $5 \%$ probabilidade.

Em relação á Tabela 3 o tratamento mombaça solteiro nas profundidades $35-40 \mathrm{~cm}, 40-$ $45 \mathrm{~cm}$ e $45-50 \mathrm{~cm}$ apresentaram resultados significativos quando adubados com o fertilizante Super fosfato triplo, tendo significância também no consórcio entre mombaça + guandu na profundidade $35-40 \mathrm{~cm}$ e $50-55 \mathrm{~cm}$, esses sistemas constataram um maior índice de compactação. Isso pode ser explicado devido o fertilizante Super fosfato triplo ter uma liberação imediata e assim não estimulando a produção de raiz em profundidade.

Tabela 3. Interação dos fatores entre as fontes de $\mathrm{P}$ com os diferentes tratamentos.

\begin{tabular}{|c|c|c|c|}
\hline \multirow{3}{*}{ Fontes de $\mathrm{P}$} & \multicolumn{3}{|c|}{ Sistema de forragens } \\
\hline & $\mathrm{M}-\mathrm{N}$ & $M+J$ & $M+G$ \\
\hline & \multicolumn{3}{|c|}{$15-20(\mathrm{~cm})$} \\
\hline$-P$ & $4,58 \mathrm{ABa}$ & $2,72 \mathrm{Aa}$ & $4,18 \mathrm{Aa}$ \\
\hline Bayóvar & $5,64 \mathrm{Bb}$ & $2,48 \mathrm{Aa}$ & 3,81Aab \\
\hline \multirow[t]{2}{*}{ SFT } & 2,19Aa & 3,35Aab & $5,01 \mathrm{Ab}$ \\
\hline & \multicolumn{3}{|c|}{$35-40(\mathrm{~cm})$} \\
\hline$-P$ & $6,59 \mathrm{Bb}$ & 3,03Aa & 3,99Aba \\
\hline Bayóvar & $4,88 \mathrm{Ab}$ & 2,13Aa & 3,31Aab \\
\hline \multirow[t]{2}{*}{ SFT } & 3,33Aa & $3,21 \mathrm{Aa}$ & $5,24 \mathrm{Bb}$ \\
\hline & \multicolumn{3}{|c|}{$40-45(\mathrm{~cm})$} \\
\hline$-P$ & $5,02 \mathrm{Bb}$ & $3,11 \mathrm{Aa}$ & 3,42Aa \\
\hline Bayóvar & $4,46 \mathrm{Bb}$ & 1,93Aa & 3,67Aa \\
\hline \multirow[t]{2}{*}{ SFT } & $3,02 \mathrm{Aa}$ & 3,07Aa & $4,55 \mathrm{Ab}$ \\
\hline & \multicolumn{3}{|c|}{$45-50(\mathrm{~cm})$} \\
\hline$-P$ & 4,97Bb & 3,17Aa & $3,16 \mathrm{Aa}$ \\
\hline Bayóvar & $3,68 \mathrm{ABb}$ & $2,00 A a$ & 3,30Aab \\
\hline \multirow[t]{2}{*}{ SFT } & 2,95Aab & 2,82Aa & $4,30 \mathrm{Ab}$ \\
\hline & \multicolumn{3}{|c|}{$50-55(\mathrm{~cm})$} \\
\hline$-P$ & $4,51 \mathrm{Bb}$ & 3,02Aa & $2,76 \mathrm{Aa}$ \\
\hline Bayóvar & $3,60 \mathrm{ABa}$ & 2,14Aa & $3,11 \mathrm{Aba}$ \\
\hline SFT & 2,96Aa & $2,48 \mathrm{Aa}$ & $4,50 \mathrm{Bb}$ \\
\hline
\end{tabular}

Médias seguidas de letras distintas (maiúscula na coluna e minúscula na linha) diferem entre si pelo teste Tukey a 5\% de probabilidade.

Os resultados apresentados na tabela 4 demonstram que não houve diferença significativa entre os SF e nem entre as fontes de P avaliadas após a saída dos animais. 
Tabela 4. Analise de compactação do solo nos sistemas de forragem (SF) e fontes de P. Presidente Bernardes-SP, saída dos animais.

\begin{tabular}{|c|c|c|c|c|c|c|c|c|c|c|c|}
\hline$S F^{(1)}$ & 2,5 & 7,5 & 12,5 & 17,5 & 22,5 & 27,5 & 32,5 & 37,5 & 42,5 & 47,5 & 52,5 \\
\hline & \multicolumn{11}{|c|}{ Profundidade $(\mathrm{cm})$} \\
\hline$M-N$ & $5,0 a$ & $7,4 a$ & $7,3 a$ & $6,9 a$ & $5,7 a$ & $5,5 a$ & $5,7 a$ & $5,7 a$ & $5,0 a$ & $4,7 a$ & $3,7 a$ \\
\hline$M+J$ & $5,7 a$ & $7,1 a$ & $6,4 a$ & $5,3 a$ & $5,2 a$ & $4,9 a$ & $4,7 a$ & $4,5 a$ & $4,5 a$ & $4,0 a$ & $3,4 a$ \\
\hline$M+G$ & $5,3 a$ & $9,2 a$ & $9,1 b$ & $6,6 a$ & $5,9 a$ & $5,6 a$ & $5,3 a$ & $5,0 a$ & $4,2 a$ & $3,7 a$ & $3,2 a$ \\
\hline \multicolumn{12}{|l|}{$\begin{array}{c}\text { Fontes de } \\
\mathbf{P}\end{array}$} \\
\hline$-P$ & $5,5 a$ & $8,8 a$ & $8,1 a$ & $7,1 \mathrm{a}$ & $6,2 a$ & $5,6 a$ & $5,2 a$ & $5,3 a$ & $4,7 a$ & $3,9 a$ & $3,1 a$ \\
\hline Bayóvar & $5,6 a$ & $7,7 a$ & $7,7 a$ & $5,8 a$ & $5,3 a$ & $5,0 a$ & $4,6 a$ & $4,6 a$ & $4,4 a$ & $4,1 a$ & $3,6 a$ \\
\hline SFT & $4,9 a$ & $7,2 a$ & $7,0 a$ & $5,9 a$ & $5,3 a$ & $5,4 a$ & $5,8 a$ & $5,3 a$ & $4,7 a$ & $4,3 a$ & $3,6 a$ \\
\hline$S F \times P$ & $\mathrm{~ns}$ & ns & ns & ns & ns & ns & ns & ns & ns & ns & ns \\
\hline $\mathrm{CV}_{\text {parcela }}$ & 29,3 & 23,0 & 16,5 & 46,8 & 45,0 & 39,9 & 28,1 & 24,6 & 28,2 & 29,9 & 21,9 \\
\hline $\mathrm{CV}_{\text {subparcela }}$ & 48,8 & 36,5 & 30,9 & 36,3 & 34,3 & 35,0 & 34,9 & 28,5 & 28,8 & 27,6 & 34,4 \\
\hline
\end{tabular}

Os resultados de matéria seca apresentados na tabela 5 indicam que na profundidade de 0 $25 \mathrm{~cm}$ não houve diferença significativa entre os SF, já na camada de $25-50 \mathrm{~cm}$ houve um maior acumulo de matéria seca de raiz no consórcio entre Mombaça + java, na profundidade de $25-50 \mathrm{~cm}$ apresentou o maior acumulo de matéria seca no consórcio entre Mombaça + guandu e no acumulo total os consórcios apresentam diferença significativa em relação ao sistema solteiro. As fontes de $\mathrm{P}$ independente da profundidade o SFT foi o que apresentou maior acumulo de matéria seca.

Tabela 5. Matéria seca de raiz de $P$. Maximum cv. Mombaça em razão do sistema de Forragens (SF) e fontes de P. Presidente Bernardes-SP.

\begin{tabular}{|c|c|c|c|c|}
\hline Tratamentos & $0-25$ & $25-50$ & $50-75$ & Acúmulo \\
\hline $\mathrm{SF}^{(1)}$ & $\mathrm{Kg} \mathrm{ha}^{-1}$ & $\mathrm{Kg} \mathrm{ha}^{-1}$ & $\mathrm{Kg} \mathrm{ha}^{-1}$ & $\mathrm{Kg} \mathrm{ha}^{-1}$ \\
\hline Mombaça - N & $1996,13 a$ & $1175,28 b$ & $884,48 b$ & $4055,89 b$ \\
\hline Mombaça + Java & $2216,52 a$ & $1403,32 a$ & $876,28 b$ & $4496,12 a b$ \\
\hline Mombaça + Guandú & $2434,85 a$ & $1067,40 \mathrm{~b}$ & $929,47 a b$ & $4431,72 a b$ \\
\hline \multicolumn{5}{|l|}{ Fontes de $\mathbf{P}$} \\
\hline$-P$ & $1963,92 b$ & $1149,39 b$ & $838,89 b$ & $3952,19 c$ \\
\hline Bayóvar & $2176,88 a b$ & $1230,80 b$ & $992,63 a b$ & $4400,31 b$ \\
\hline SFT & $2466,08 a$ & $1406,86 a$ & $1052,75 a$ & $4925,68 a$ \\
\hline $\begin{array}{c}\text { Interação SF x Fontes } \\
\text { de P }\end{array}$ & ns & ns & ns & ns \\
\hline $\mathrm{CV}_{\text {parcela }}(\%)$ & 16,93 & 8,10 & 20,68 & 10,36 \\
\hline $\mathrm{CV}_{\text {subparcela }}(\%)$ & 15,56 & 15,58 & 19,98 & 9,63 \\
\hline
\end{tabular}

Médias seguidas de letras distintas na coluna, dentro do fator SF e fontes de P, diferem entre si pelo teste Tukey a $5 \%$ probabilidade.

A utilização de diferentes sistemas de cultivos de pastagem associada com distintas fontes de adubações fosfatadas apresentam resultados que devem ser estudados por maior período de tempo, para que com isso tenhamos uma maior clareza.

\section{DISCUSSÃO}

Segundo Herrick \& Jones (2002), o penetrômetro de impacto registra o RP máximo por uma unidade de profundidade. Em vários estudos mostra que a compactação está associada á taxa de lotação animal, sendo que quando a carga animal é ajustada da forma correta a compactação é registrada apenas nas camadas superficiais, com isso ao longo do tempo o solo volta a sua condição inicial (CONTE et al., 2011; SILVA et al., 2014; BONETTI et al., 2015). 
A compactação do solo vai depender de fatores, principalmente, da classe de solo, do teor de umidade contido, da taxa de lotação animal, da espécie de forragem a ser utilizada no sistema e seu respectivo teor de massa produzida (MARCHÃO et al., 2007). Em estudos feitos por Spera et al. (2004) e Marchão et al. (2007) foi observado que a compactação resultante do pisoteio animal não teve interferência negativa em que desrespeito a resistência á penetração, entretanto na área de pastejo houve uma aumento significativo na densidade do solo.

Contudo não podemos deixar de ressaltar que grande parte das avaliações de resistência do solo á penetração disponível na literatura é de solos argilosos, contendo pouca informação sobre solos arenosos consorciados entre gramíneas e leguminosas forrageiras.

\section{CONCLUSÃO}

No presente trabalho observou um efeito maior de compactação nas profundidades abaixo de $35 \mathrm{~cm}$ independente do sistema de forragem e fonte de fósforo. A entrada dos animais não influenciou na compactação do solo. Os consórcios apresentaram maior produção de raiz juntamente com o super fosfato triplo

\section{REFERENCIAS}

ANUALPEC. Anuário da Pecuária Brasileira. São Paulo: Instituto FNP, 360p, 2014.

AZEVEDO, R.L.; RIBEIRO, G. T.; AZEVEDO, C. L. L. Feijão Guandu: Uma Planta Multiuso. Revista da Fapese, v.3, n.2, p. 81-86 jul./dez. 2007.

BONETTI, J. de A.; PAULINO, H.B.; SOUZA, E.D. de; CARNEIRO, M.A.C.; SILVA, G.N. da. Influência do sistema integrado de produção agropecuária no solo e na produtividade de soja e braquiária. Pesquisa Agropecuária Tropical, v.45, p.104-112, 2015. https://doi.org/10.1590/1983$\underline{40632015 \mathrm{v} 4529625}$

CARVALHO, G. G. P. E PIRES, A. J. V. Leguminosas tropicais herbáceas em associação com Pastagens. Archivos de zootecnia vol. 57(R), p. 104.2008.

CECATO, U; PEREIRA, L. A. F.; JOBIM, C. C. Influência das adubações nitrogenadas e fosfatadas sobre a composição químico-bromatológica do capim-Marandu (Brachiaria brizantha) (Hochst) Stapf cv. Marandu). Acta Scientiarum, Maringá, v. 26, n. 3, p. 409-416, 2004. https://doi.org/10.4025/actascianimsci.v26i3.1837

CONTE, O.; FLORES, J.P.C.; CASSOL, L.C.; ANGHINONI, I.; CARVALHO, P.C. de F.; LEVIEN, R.; WESP, C. de L. Evolução de atributos físicos de solo em sistema de integração lavoura-pecuária. Pesquisa Agropecuária Brasileira, v.46, p.1301-1309, 2011. https://doi.org/10.1590/S0100$\underline{204 \times 2011001000026}$

EMBRAPA. Centro Nacional e Pesquisa em Solos. Sistema Brasileiro de Classificação de Solos. Brasília: Embrapa-SPI; Rio de Janeiro: Embrapa-Solos, p.306, 2006.

FERREIRA, D. F. Análise estatística por meio do SISVAR para Windows versão 4.0. In: Reunião anual da região brasileira da sociedade internacional de biometria. São Carlos. Anais: UFSCar, p. 255-258, 2000.

HERRICK, J.E.; JONES, T.L. A dynamic cone penetrometer for measuring soil penetration resistance. Soil Science Society of America Journal, Madison, v.66, n.4, p.1320-4, 2002. 
Marchão RL, Balbino LC, Silva EM, Santos Junior JDG, Sá MAC, Vilela L, Becquer T. Qualidade física de um Latossolo Vermelho sob sistemas de integração lavoura pecuária no Cerrado. Pesq Agropec Bras. p.873-82, 2007.

RAIJ, B. van; ANDRADE, J.C.; CANTARELLA, H.; QUAGGIO, J.A. Análise química para avaliação da fertilidade de solos tropicais. Campinas: Instituto Agronômico, 2001. 284p.

SPERA, S. T.; SANTOS, H. P.; FONTANELI, R. S.; TOMM, G. O. Efeito de sistemas de produção de grãos envolvendo pastagens sob plantio direto nos atributos físicos de solo e na produtividade. Revista Brasileira de Ciência do Solo, v. 28, p. 533-542, 2004. https://doi.org/10.1590/S0100$\underline{06832004000300014}$

SILVA, F.D. da; AMADO, T.J.C.; FERREIRA, A.O.; ASSMANN, J.M.; ANGHINONI, I.; CARVALHO, P.C. de $F$. Soil carbon indices as affected by 10 years of integrated crop-livestock production with different pasture grazing intensities in Southern Brazil. Agriculture, Ecosystems and Environment, v.190, 60-68, 2014. DOI: 10.1016/j.agee.2013.12.005.

TORMENA, C.A.; ROLOFF, G. Dinâmica da resistência à penetração de um solo sob plantio direto. Revista Brasileira de Ciência do Solo, Campinas, v.20, n.2, p.333-39, 1996. 